
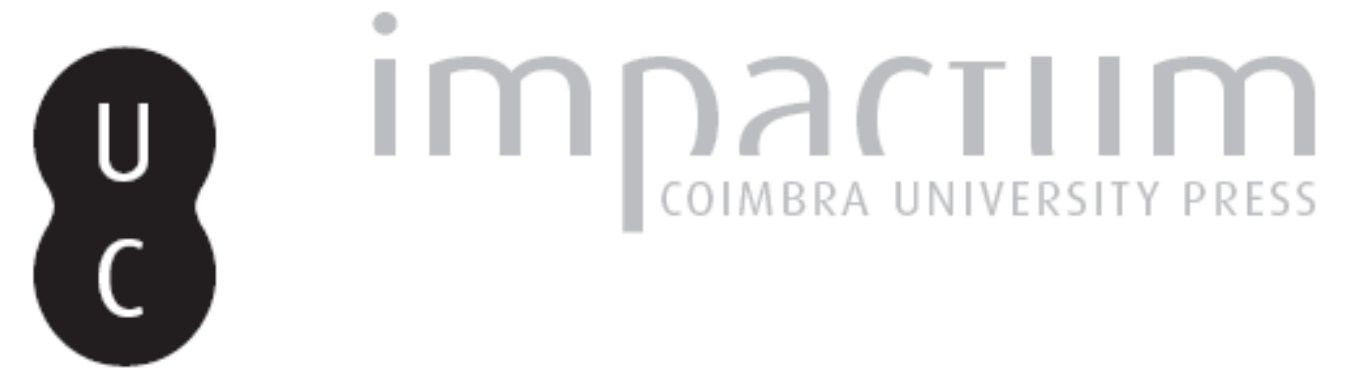

À propos d'une représentation de villa sur une mosaïque de Tolède: (Espagne)

Autor(es): $\quad$ Gorges, Jean-Gérard

Publicado por: Imprensa da Universidade de Coimbra

URL persistente:

URI:http://hdl.handle.net/10316.2/45617

DOI:

DOI:https://dx.doi.org/10.14195/1647-8657_25_11

Accessed : $\quad$ 26-Apr-2023 15:31:38

A navegação consulta e descarregamento dos títulos inseridos nas Bibliotecas Digitais UC Digitalis, UC Pombalina e UC Impactum, pressupõem a aceitação plena e sem reservas dos Termos e Condições de Uso destas Bibliotecas Digitais, disponíveis em https://digitalis.uc.pt/pt-pt/termos.

Conforme exposto nos referidos Termos e Condições de Uso, o descarregamento de títulos de acesso restrito requer uma licença válida de autorização devendo o utilizador aceder ao(s) documento(s) a partir de um endereço de IP da instituição detentora da supramencionada licença.

Ao utilizador é apenas permitido o descarregamento para uso pessoal, pelo que o emprego do(s) título(s) descarregado(s) para outro fim, designadamente comercial, carece de autorização do respetivo autor ou editor da obra.

Na medida em que todas as obras da UC Digitalis se encontram protegidas pelo Código do Direito de Autor e Direitos Conexos e demais legislação aplicável, toda a cópia, parcial ou total, deste documento, nos casos em que é legalmente admitida, deverá conter ou fazer-se acompanhar por este aviso.

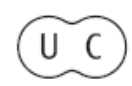


FACULDADE DE LETRAS

INSTITUTO DE ARQUEOLOGIA

CONIMBRIGA

$V O L U M E X X V$

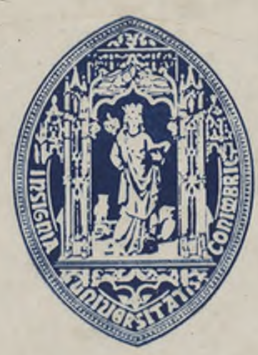

UNIVERSIDADE DE COIMBRA

1986 
S R R A D GORGE

G.N.R.S., Maison des Pays Ibériques

\section{À PROPOS D'UNE REPRÉSENTATION DE VILLA SUR UNE MOSAÏQUE DE TOLÈDE (ESPAGNE)}

«Conimbriga», XXV (1986), p. 175-192

Resum : As representações de villae em mosaicos romanos são pouco

numerosas, localizando-se principalmente na África do Norte. A Península Ibérica, todavia, fornece alguns exemplos deste tipo iconográfico, muito apreciado pelos grandes proprietários fundiários do Baixo Império. Entre eles, o pavimento da «Vega Baja» de Toledo, durante muito tempo mal interpretado, é tanto mais precioso quanto é o único da península a representar os diferentes aspectos arquitectónicos de um domínio rural do século iv, propondo uma série de questões sobre a leitura destas representações, o seu significado e o seu grau de relação com o real.

RÉsumé: Les représentations de villae sur des mosaïques romaines sont peu nombreuses et principalement localisées en Afrique du Nord. La péninsule ibérique, toutefois, fournit quelques exemples de ce type iconographique, très prisé des grands propriétaires terriens du Bas-Empire. Parmi eux, le pavement de la «Vega Baja» de Tolède, longtemps mal lu, est d'autant plus précieux qu'il est le seul pour la péninsule à présenter les différents aspects architecturaux d'un domaine rural du IVe siècle. Ainsi se trouve posée une série de questions sur la lecture de ces représentations, leur signification et leur degré de rapport avec le réel. 
(Página deixada propositadamente em branco) 


\section{A PROPOS D'UNE REPRÉSENTATION DE VILLA SUR UNE MOSAÏQUE DE TOLÈDE (ESPAGNE)}

Un livre relativement récent $\left({ }^{1}\right)$ a mis en valeur, voici quelques années, à travers l'exemple des représentations de villas sur les mosaïques africaines tardives, l'intérêt socio-économique et documentaire de telles oeuvres, de même que les règles de perspectives et de figuration auxquelles elles obéissent. Par un catalogue des sources et l'analyse iconographique systématique des représentations architecturales connues, l'auteur a su dégager les lois présidant à la composition des tableaux, à la détermination des proportions, des plans et des effets de rendu, au choix des couleurs. Il a montré combien la façade de l'édifice peut être valorisée en tant que figuration synthétique de la villa, et par là même combien le rôle prépondérant accordé aux différents éléments architectoniques retenus pour la composer peut être significatif. Il a aussi démontré combien ce type de représentation de l'architecture rurale pouvait à la fois être général et mordre sur le concret.

Or, il se trouve, dans cette ligne de préoccupation, qu'une mosaïque provenant d'une villa suburbaine de Tolède invite à réfléchir, pour la péninsule ibérique, à ce type de représentation figurée. Il s'agit d'un pavement relativement connu par ailleurs $\left({ }^{2}\right)$,

l1) s а п о ш ск (т.), Les représentations de villas sur les mosaïques africaines tardives (Archiwum Filologiczne, XXXVII), Varsovie, 19;8.

(2) Melida (J. R.), El arte en España durante la época romana, dans Historia de España, dirigida por R. Menéndez Pidal, II, España: Romana, Madrid, 1935, p. 710-713; san roman fernandez (F. de B.), El segundo mosaico romano de la Vega Baja de Toledo, dans Anuario 
exposé au Musée Santa Cruz de Tolède, catalogué sous l'appellation banale de «Mosaïque des Quatre Saisons», ou encore de «Mosaïque aux poissons», du fait de l'existence d'un emblema central consacré à ce thème.

\section{Historique}

La découverte du pavement remonte au mois de février 1923, lorsqu'à l'occasion de travaux d'agrandissement effectués dans l'ancienne manufacture d'armes blanches de la «vega baja» de Tolède, à deux kilomètres au nord-ouest de la ville et à proximité du Tage, on mit au jour, par trois mètres de profondeur, les traces éloquentes d'une luxueuse villa romaine. La mosaïque qui nous intéresse, incomplète $(6,20 \mathrm{~m} \times 3,40 \mathrm{~m})$, tapissait le sol d'une salle dont les dimensions totales avoisinaient 40 mètres carrés, et dans laquelle Mélida reconnut, selon toute vraisemblance avec raison, le triclinium de l'établissement ( ${ }^{3}$ ). La disposition en emblema du pavement figuré (PI. I, Fig. 1), entouré de tapis rectangulaires à motifs géométriques simples sur au moins trois côtés (le quatrième a disparu), et les traces d'une fontaine à proximité du seuil concourent à cette identification. Une restitution dessinée du pavement $\left(^{4}\right)$

del Cuerpo Facultativo de Archiveros, Bibliotecarios y Arqué́logos ( - Homenaje Mélida, III), Madrid, 1934, p. 340-342; aragoneses (J.), Museo Arqueológico de Toledo (Guía de los Museos de España, VII), Madrid, 1958, p. 71-72; в ваzquez (J. M.a), Mosaicos romanos de la Real Academia de la Historia, Ciudad Real, Toledo y Cuenca (Corpus de Mosaicos de España, V), Madrid, CSIC, 1982, p. 33-40 et pl. 19-23, adopte quant à lui l'appellation de «mosaïque aux poissons».

(3) Melida (J. R.), Nuevas relaciones del gran Toledo: el maravilloso mosaico romano de la Fábrica de armas, dans Toledo, Revista de Arte, IX, 199, 1923, p. 734-735; Idem, Un mosaico descubierto en Toledo, dans Boletín de la Real Academia de la Historia, LXXXIII, 1923, p. 19-23 (stt. p. 22); Idem, Informe acerca de un mosaico romano descubierto en Toledo, dans Boletín de la Real Academia: de Bellas Artes de San Fernando, XVI, 1923, p. 132-135.

$\left({ }^{4}\right)$ aragoneses (J.), Museo Arqueológico de Toledo (Op. cit.), Madrid, 1958, pl. III. 
fait d'ailleurs clairement ressortir l'ordonnance et la variété des panneaux qui composaient le sol de cette salle à manger.

Sectionnée sur l'un de ses côtés par un mur tardif, la partie centrale (à l'origine un carré de $2,48 \mathrm{~m}$ de côté), délimitée par un cadre composé d'une théorie de triangles tangents, offrait en particulier un médaillon central d'inspiration marine (poissons, fruits de mer, mollusques, crustacés...), représentation de la mer et de sa faune. Incluse dans un réseau compliqué de tresses, le dessin en buste des Quatre Saisons personnifiées donna au pavement sa dénomination originale. Rapidement publiée ${ }^{5}$ ), la mosaïque fut tout d'abord conservée sur place. Par la suite, gravement endommagée par des infiltrations provoquées par les remontées de la nappe phréatique du fleuve tout proche, on la transféra finalement en 1932 au musée archéologique Santa Cruz où, après restauration, elle est encore actuellement conservée. Les travaux de dépose fournirent l'occasion de redégager partiellement un secteur contigu interprété par Mélida comme le péristyle de la villa, livrant une seconde mosaïque figurée, déjà aperçue en 1923, et qui venait orner le fond d'un bassin octogonal (PI. I, Fig. 3). Son décor de scènes de pêche, de bateaux, de paysages portuaires maritimes ou fluviaux, mêlant de nombreux éléments d'architecture, a été longuement repris et commenté par A. Balil $\left(^{6}\right)$, qui note la fréquence en Afrique du Nord de ces thèmes inspirés des représentations marines hellénistiques et alexandrines. Ce sont là des images nautiques juxtaposées, où la mer l'emporte sur le fleuve, mais qui se rattachent autant au réel par leur précision qu'à la tradition du paysage idyllique par leur agencement. Néanmoins, la profusion des vaisseaux marchands, des installations portuaires, l'abondance des scènes de pêche incitent à y voir un rappel insistant des activités commerciales et économiques liées au domaine aquatique.

La mosaïque des Quatre Saisons, en revanche, n'a pas donné lieu à une véritable étude, particulièrement en ce qui concerne

(5) Cf. note 3 .

(') в в циц (A.), Mosaico con escenas portuarias hallado en Toledo, dans Homenaje al Profesor C. de Mergelina, Murcie, 1961-1962, p. 123-138. 
ses éléments architecturaux figurés $\left({ }^{7}\right)$. Or, il se trouve que ce pavement fait problème, car il regroupe a priori des thèmes qui ne paraissent avoir aucun rapport entre eux. Ainsi, la composition décorative, formée d'un octogone central (PI. I, Fig. 2) et d'un réseau de tresse tangent délimitant de petits tableaux de formes variées (carrés, demi-cercles, éventails, etc.), présente à la fois une iconographie des Saisons, un emblema circulaire d'une rare qualité consacré à la faune marine, des tableaux mettant en scène des représentations architecturales.

\section{Lecture et Chronologie}

Considérons tout d'abord le schéma de construction de la mosaïque dite des Quatre Saisons (PL I; Fig. 1 et 2). La thématique générale du pavement mêle effectivement au moins deux sources d'inspiration: l'une temporelle et rurale, qui s'illustre particulièrement par les représentations des saisons accompagnées chacune d'éléments caractéristiques empruntés à la flore ou à la faune (PI. I, Fig. 1, secteurs 1 à 4); l'autre aquatique, qui s'exprime avec force à travers le magnifique médaillon central (PL I, Fig. 2, E). Toutefois, un examen attentif du pavement montre que ces deux thèmes sont étroitement liés, et qu'ils servent de toile de fond à un troisième registre, celui qui, sans aucun doute, était le plus important aux yeux du maître de maison, et que l'artiste a disposé habilement en demi-cercles tangents aux quatre sommets de l'octogone qui occupe à lui seul plus du tiers du champ figuratif. $\mathrm{Du}$ reste, ce sont ces mêmes motifs qui viennent s'intercaler têtebêche entre chacune des saisons, lesquelles semblent rythmer le déroulement des scènes figurées. Il nous faut donc examiner, dans un premier temps, les différents registres de la mosaïque.

$\left(^{7}\right) \quad$ On trouvera dans les descriptions rapides de J. M. ${ }^{\text {a }}$ в azquez, Mosaicos romanos... (Op. cit.), p. 36-40, les parallèles connus pour chacun des motifs figurés, sans que soit proposée toutefois une interprétation d'ensemble des décors ou du pavement. 


\section{a) Le registre des saisons}

Placées en écoinçons et regardant à l'extérieur du pavement dont elles commandent la construction, les saisons battent la mesure du temps campagnard ( $\left.{ }^{8}\right)$, mais aussi plus généralement de l'ensemble des activités humaines. Quatre jeunes femmes symbolisent les quatre grandes divisions de l'année, accompagnées chacune de divers éléments figurés:

- Printemps (1): buste couronné de fleurs; en association, paniers ou boisseaux de fleurs, fleurs isolées, rameaux de laurier.

- Été (2): figure entourée d'épis de céréales; autour d'elle: gerbes de blé, épis, faucille (tesselles bleues en pâte de verre).

- Automne (3): buste couronné de pampres; en attribut: grappes de raisin, branches de figuiers et de châtaigniers.

- Hiver (4) : buste de femme dont la tête est couverte ; les mains tiennent une tige de roseau et un rameau d'olivier, dans les cadres attenants: canards, pigeons, chardons, rameaux d'olivier.

\section{b) Le registre de l'octogone}

Deux éléments principaux doivent retenir l'attention, si l'on excepte les motifs décoratifs à thèmes géométriques. Il s'agit en premier lieu de la grande guirlande végétale qui enserre Vemblema central, et dont les éléments constitutifs, fruits et végétaux, ne sont pas disposés au hasard, mais bien en regard des quatre saisons correspondantes, placées aux angles (PL I, Fig. 1 et Fig. 2, F). Les pommes et les fruits secs (amandes, noix, figues) de l'Hiver sont séparés par un ruban de fleurs printanières, de même que les épis de l'Été, ou encore les grappes et les pampres de l'Automne. C'est là un détail important, bien noté par Mélida $\left({ }^{9}\right)$, et dont l'intérêt est de montrer la rigoureuse construction de l'ensemble du pave-

${ }^{8}$ ) Gorges (J.-G.), Les villas hispano-romaines. Inventaire et problématique archéologiques (Publication du Centre Pierre Paris, 4), Paris, 1979, p. 155.

( $\left.{ }^{9}\right)$ Melida (J. R.), Un mosaico descubierto en Toledo, (Op. cit.), 1923, p. 20. 
ment. L'emblema circulaire central (PL I, Fig. 2), avec sa thématique marine, n'en tranche que davantage, encore individualisé par la richesse de sa polychromie en pâte de verre et l'extrême finesse des tesselles utilisées par le mosaïste (jusqu'à trois millimètres de côté ). Notons cependant qu'il est entouré d'une double ligne de postes (l'une circulaire, l'autre octogonale), symboliques images des flots qui constituent autant de rappels de l'inspiration marine. Il est clair qu'elles répondent au médaillon central de la même façon que la tresse végétale répondait aux saisons. La composition de l'ensemble du pavement figuré apparaît réfléchie et les quatre grandes divisions de l'année ne rythment pas seulement les activités rurales, mais bien aussi les activités maritimes.

\section{c) Le registre des représentations architecturales}

De la même main que la totalité de la mosaïque (excepté 1 'emblema marin, sans doute importé), les quatre tableaux demicirculaires qui composent ce troisième registre revêtent une importance toute particulière, non à cause de leur taille qui demeure modeste (33 centimètres de diamètre à la base), mais bien parce qu'ils sont, pour la péninsule ibérique, l'unique représentation en volets des divers éléments constitutifs d'un domaine rural $\left({ }^{10}\right)$. Observés séparément par Mélida, qui voyait dans la construction

(10) La mosaique d'Arroniz, bien connue, offre dans une composition polygonale neuf tableaux trapézoïdaux séparés par des tresses représentant chacun l'une des Neuf Muses avec ses attributs traditionnels. Le centre est occupé par un médaillon circulaire figurant un groupe équestre. Chaque muse est accompagnée d'un dominus, avec, en arrière-plan, une représentation de villa dans un décor à la végétation typiquement africaine. $\mathrm{Si}$ un parallèle entre les deux pavements ne serait pas sans intérêt, on se bornera ici à constater que la mosaïque d'Arroniz privilégie les représentations architecturales de villas seigneuriales et les multiplie, ôtant par là tout caractère documentaire précis. Sur cette mosaïque, voir en particulier: a. fernandez de aviles, El mosaico de las Musas de Arroniz y su restauración en el Museo Arqueológico Nacional, dans A.E.A., XVIII, 1945, ९. 342-350 + planches. 
principale un théâtre avec ses gradins et ses colonnades $\left.{ }^{\mathrm{n}}\right)$, ces tableaux n'ont effectivement jamais été sentis comme une suite logique illustrant un même ensemble. Il suffit pourtant de les considérer tour à tour pour en être convaincu.

A. Placée entre les figurations de l'Hiver et du Printemps, visible depuis la porte d'entrée, la représentation d'une villa urbana tient la place d'honneur (PI. II, Fig. 1). Il s'agit, à première vue, d'une villa linéaire à galerie de façade et tours d'angle. Les colonnes, qui reposent sur un stylobate haut composé de trois rangées de tesselles, sont au nombre de huit, et la galerie ne semble pas présenter d'accès direct. Elle vient au contraire aboutir à chaque extrémité à deux pavillons d'angle, parfaitement symétriques; le mosaïste les a représentés sur deux plans, à la fois de face et de profil, sans souci apparent de la vraisemblance puisque les deux flancs extérieurs des tours sont tous les deux montrés. Ce sont des bâtiments importants, dotés d'une porte en façade (tesselles noires disposées en $\mathrm{T}$ ) et forts de deux étages supérieurs, délimités chacun par une rangée de tesselles noires symbolisant les poutres de plafond qui soutiennent habituellement les planchers (PI. IV, Fig. 1). Curieusement, alors que ce n'est pas le cas des bâtiments figurés sur la mosaïque octogonale du bassin voisin (PI. III, Fig. 2, A et B), les fenêtres ne sont pas représentées. Elles ne le sont du reste sur aucune construction de la mosaïque dite «des Quatre Saisons». Ornés chacun d'un fronton richement décoré (tesselles bleues en pâte de verre), munis d'acrotères, les deux corps d'angle sont recouverts d'une toiture de tegulae et d'imbrices, l'artiste ayant employé le brun clair pour figurer les tuiles plates et le marron ou le noir pour rendre les faitières et les couvre-joints, se réservant la couleur rouge pour mieux rendre, dans les entre-colonnements, le pourpre habituellement employé pour enduire les stucs muraux de la plupart des villas du Bas Empire.

Entourée de vertes frondaisons à l'extérieur, la galerie portiquée en demi-cercle, qui offre douze colonnes bien visibles, représente à l'évidence le péristyle que s'est adjoint ce riche établisse-

(u) Op. cit., 1923, p. 21. 
ment seigneurial. La technique de représentation est absolument semblable à celle qui a été utilisée pour la galerie de façade, et si Ton connaît quelques exemples de villas possédant une galerie en hémicycle ( $\left.{ }^{12}\right)$, la figuration en élévation et en demi-cercle d'un ensemble architectural orthogonal caché peut aussi trouver de nombreux parallèles. On pourrait citer une représentation identique de villa à péristyle sur un plat d'argent ciselé de Rome ( $\left.{ }^{13}\right)$, ou encore plus simplement le dessin semi-circulaire des murailles d'Emerita sur les monnaies autonomes de cette colonie (PI. IV, Fig. 3 et 4). Elles montrent d'ailleurs l'ancienneté relative de ce procédé, qui trouve vraisemblablement sa source dans le souci d'utiliser au mieux le champ spatial délimité par la configuration d'un cadre circulaire ou semi-circulaire.

Enfin, à l'intérieur du péristyle ainsi défini, des tesselles bleues disposées en ligne sur un fond blanc neutre suggèrent la circulation de l'eau dans de multiples canaux et bassins. Villa et jardin attenant sont protégés par un mur d'enceinte représenté en coupe et dont on a voulu également montrer la porte monumentale, figurée en T (PI. II, Fig. 1).

B. Situé entre l'Automne et l'Hiver, le panneau B (PI. II, Fig. 2) est le second par ordre d'importance, ce qui explique sa situation face au triclinium destiné à recevoir les invités que le maître de maison veut particulièrement honorer. Il est vrai que l'on peut y contempler, entre deux petits bâtiments très soignés, une vue des jardins de la villa, symbolisés par un arbre de type idyllique élégamment dessiné (14), et qui abritent un personnage assis trônant en majesté en compagnie de trois autres figures en pied. Le dominus, car il ne peut s'agir que de lui, bénéficie en outre d'un traitement en polychromie, alors que les trois

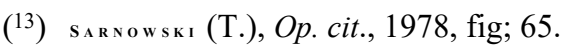

${ }^{(14)}$ Voir g rıма (P.), Les Jardins Romains, 2. a éd. P.U.F., Paris, 1969.

(12) mansuelu (G. A.), Urbanistica e architectura della Cisalpina romana, 2 vol. ( = Latomus, 111), Bruxelles, 1971, pi. LXXIV, C et Cl; fernandez castro (м.а C.), Las llamadas «Termas》 de Rielves (Toledo), dans «Archivo Español de Arqueología», 50-51, 1977-1978, p. 209-252, fig. 3 et 4 . 
silhouettes d'allure féminine qui se trouvent à ses côtés sont simplement représentées par des tesselles uniformément brunjaune. Nous serions tenté de voir dans cette scène une représentation du maitre de maison et de sa famille, dans un lieu qui leur tient particulièrement à coeur. La construction de gauche, en effet, semble bien devoir être interprétée comme un petit temple privé. L'édifice, sans étage apparent, présente un fronton richement décoré (tesselles en pâte de verre) surmonté d'un acrotère particulièremente visible. Surtout, sa façade offre à chaque angle, dessinées par une double rangée de tesselles jaunes, deux colonnes ou pilastres avec bases et chapiteaux. Enfin, sur un fond neutre, l'artiste a représenté debout, sur un large autel de tesselles rouges, la silhouette d'une statue qui semblerait de bronze, image de la divinité abritée par l'édifice et dont l'allure générale paraît indiquer le mouvement (PI. IV, Fig. 2). Priape des jardins ou Mercure surmonté d'une coiffe, ou encore autre divinité, l'identification n'est guère aisée. L'absence de toute colonnade en façade trahit un bâtiment plus modeste, lui aussi sans étage. Son sol cimenté ou pavé a toutefois donné lieu à une figuration spéciale (une ligne de tesselles rouges) et la largeur de la porte (deux rangées de tesselles noires en T) rappelle au spectateur l'échelle réelle d'une construction dans laquelle il ne faut voir sans doute qu'un pavillon de jardin, le tout étant clos, là encore, par un mur d'enceinte bien visible dans l'angle inférieur droit du panneau.

C. Le tableau suivant (PI. II, Fig. 3) est malheureusement incomplet, sectionné à son sommet par un mur tardif venu amputer d'une large part l'ensemble des pavements du triclinium. Toutefois, ce dommage ne nuit que partiellement à l'interprétation que l'on peut en donner, surtout si l'on a recours aux clichés les plus anciens, les restaurations successives n'ayant fait qu'accentuer la détérioration de cette zone, au point de la faire quasiment disparaître. Entourées d'un mur d'enceinte figuré en coupe à chaque extrémité du panneau semi-circulaire, les trois constructions représentées illustrent sans aucun doute la pars rustica d'un établissement campagnard. De part et d'autre d'une construction cylindrique (grenier à grain, pigeonnier?) deux bâtiments avec portes en $\mathrm{T}$ et respectivement deux et un étages symbolisent les dépendances 
rustiques du domaine; deux troncs d'arbre encore visibles indiquent au spectateur qu'elles étaient établies dans une cour plantée.

L'absence de fenêtres apparentes ne signifie pas que l'on soit obligatoirement en présence de constructions non habitables. Les fenêtres, on l'a vu, ne sont pas non plus représentées sur les corps d'angle de la villa qui occupe le panneau A, ni sur aucune autre construction appartenant au pavement des Quatre Saisons. C'est là une particularité technique qui différencie radicalement cette mosaïque de celle du bassin du péristyle où les représentations architecturales ne manquent jamais de mentionner, par le biais d'une tesselle noire, l'existence d'une ouverture sur le jour. On peut voir, dans les deux édifices rectangulaires recouverts d'une toiture de tegulae et d'imbrices, des locaux mixtes servant aussi bien à l'habitation du personnel dans les étages supérieurs qu'à l'entrepôt du matériel et des récoltes, ou à l'abri du bétail, au niveau inférieur.

D. Ainsi, maison de maître, jardin idylique, pavillon de luxe, temple privé et pars rustica composent en un triptyque une vue d'ensemble d'un riche domaine campagnard. Elle serait pourtant incomplète si l'on omettait de rattacher à cette peinture un quatrième volet, dont l'interprétation a toujours été bien délicate $\left({ }^{15}\right)$ (PI. III, Fig. 1). Placé entre l'Été et l'Automne, peut-être comme le notait Mélida par référence à l'équinoxe $\left({ }^{16}\right)$, le dernier panneau nous ramène au thème de l'eau. La description donnée, toutefois, n'est pas satisfaisante, bien que la valeur symbolique $\mathrm{du}$ tableau soit facilement explicable. Tour de surveillance à toit plat ou plutôt phare placé à l'extrémité d'une digue ou jetée perpendiculaire à l'observateur, le motif architectural sépare en deux registres le champ de ce dernier volet. A droite, le dessin d'un gouvernail de bateau occupe la majorité de l'espace, en même temps qu'un dauphin, dont la tête est aujourd'hui incomplète, émerge des flots. A gauche, on peut reconnaître la figuration complète d'une

(15) Blazquez (J. M.a), Mosaicos romanos (Op. cit.), Madrid, 1982, p. 38, n'en donne encore qu'une interprétation confuse et partiellement inexacte.

(16) Op. cit., 1923, p. 21. 
poupe en col de cygne (et non un monstre marin!), ainsi qu'une tête humaine sortant de l'eau. Il ne saurait s'agir de celle d'un naufragé, comme le suggère Mélida, mais bien de la tête d'un nageur, pendant logique de la tête de dauphin apparaissant dans le premier registre. De la même façon se répondent ces deux symboles de la navigation marchande que sont le gouvernail et l'aplustre en col de cygne, que l'on retrouve précisément associés sous la forme d'un trophée dans la mosaïque du bassin (PI. III, Fig. 2).

L'évocation de l'eau et des activités de transport liées à la batellerie ou à la navigation ne saurait surprendre dans le cadre d'un riche établissement rural placé sur les bords même du Tage. En ce sens, ce quatrième médaillon appartient bien, comme les trois autres, à la même série descriptive d'un domaine seigneurial, telle que l'a voulue un propriétaire hispano-romain de l'époque de la Tétrarchie ou du début du IVe siècle après Jésus-Christ, époque proposée par Balil pour la confection de ces pavements $\left({ }^{17}\right)$, les datations antérieures n'étant plus aujourd'hui acceptables. Bien plus, par son allusion volontaire au commerce maritime ou fluvial, ce dernier tableau rejoint et explique $Y$ emblema central dont la faune marine pouvait à première vue paraître déplacée ou ne relever que d'une illustration des plaisirs de la table. Rythmant le temps des campagnes, mais aussi celui de la navigation, les saisons s'intégrent parfaitement dans une iconographie homogène qui fait ressortir combien la mosaïque du triclinium et celle du bassin du péristyle sont liées dans leur esprit et dans leur thématique, expressions certes d'un goût culturel, mais aussi de la volonté d'un homme qui, pour mieux faire ressortir cette association désirée, est allé jusqu'à porter son choix sur deux compositions octogonales.

(17) Balil (A.), Mosaico con escenas portuarias... (Op. cit.), 1961-1962, p. 137-138; cette datation est d'ailleurs reprise par A. Blazquez, Op. cit., 1982, p. 36. 


\section{I I. L'art des latifundiaires: programmes, conventions et expres- sions}

La lecture du pavement de Tolède telle que nous venons de l'effectuer n'est pas sans poser bien des questions. Elles sont principalement de deux ordres: on peut en effet se pencher sur l'analyse détaillée des représentations et s'interroger sur la mesure dans laquelle elles permettent la reconstitution des constructions réelles; on peut aussi chercher à découvrir leur contenu sémantique et leurs liens avec un programme iconographique révélateur de mentalités nouvelles.

Placée entre l'Hiver et le Printemps, l'image de la villa urbana semble ouvrir la succession des tableaux. Elle illustre de façon remarquable les règles de la représentation architecturale définies par T. Sarnowski. La pars urbana apparaît en effet dans un plan unique, sans que l'artiste ait recherché le moins du monde à produire un effet de profondeur spatiale. Bien au contraire, il s'est efforcé, dans une «perspective aplanie», de montrer sur un même plan plusieurs faces du bâtiment pris pour modèle: ainsi, la galerie à colonne est représentée de face dans sa totalité, mais la ligne partielle de tesselles brunes qui la surmonte (PI. IV, Fig. 1) semble indiquer un changement de direction à chacune de ses extrémités, la galerie continuant en fait en retour sur les faces internes des pavillons d'angle. Ces derniers sont figurés «de biais», leur flanc extérieur étant directement accolé à la façade, sans le souci de rechercher une ligne de fuite vraisemblable. L'ensemble donne l'impression d'être contemplé en une seule fois sous plusieurs angles de vue, impression renforcé e par le procédé d'étagement utilisé pour rendre visible le péristyle de la villa, normalement caché derrière la façade.

L'importance primordiale accordée aux représentations de galeries (façade, péristyle) se manifeste dans les proportions qui leur sont attribuées, en même temps qu'elles trahissent l'existence d'une hiérarchisation entre les différents éléments constitutifs de la villa. Ceux-ci, du reste, ont fait l'objet d'un choix, et il est aisé de faire l'inventaire des éléments architecturaux signifiants retenus par le mosaïste. Ce sont d'une part les galeries, avec colonnades et stylobates, et d'autre part les pavillons d'angle, 
dont il a voulu marquer les portes, les étages, les frontons munis d'acrotères et les toitures de tuiles plates. La stylisation de certains éléments fait appel à des traits d'observation: la division en étages est signalé par une ligne simple indiquant les têtes de poutres de plafond pendant que les portes des tours sont schématisées par un $\mathrm{T}$ rappelant les joints centraux et supérieurs d'un système d'ouverture à double battant. Les fenêtres sont omises, mais les tours sont agrémentées de faiteaux en forme de triangles ou de cônes, comme il est fréquent d'en relever dans les représentations de palais et d'églises basilicales de la basse antiquité et du moyenâge $\left({ }^{18}\right)$.

Dans l'expression du rendu architectural, les couleurs ne sont pas non plus à négliger, car elles paraissent correspondre à un code précis de représentation. Ici, le noir souligne les détails architecturaux; les colonnes (de marbre?) sont rendues, comme sur toutes les mosaïques africaines recensées par Sarnowski, au moyen d'une ligne de cubes blancs; des tesselles bleues en pâte de verre soulignent la* richesse présumée de la décoration des frontons. Il n'est pas jusqu'à la couleur jaune-brun employée pour la coverture des toits qui ne puisse avoir sa signification: distinction d'avec les tesselles rouges utilisées pour former le fond des galeries ou bien représentation de dalles de marbre dorées ou de tuiles peintes? Enfin, une ligne de tesselles de couleur différente peut aussi marquer, dans le plan unique qui est celui du dessin sur mosaïque, un changement d'orientation dans le plan réel; c'est le cas pour la ligne de tesselles grises qui, sur chacun des pavillons, marque l'angle de la façade avec le côté extérieur.

Si l'on admet, avec T. Sarnowski, que le motif de la villa à galerie et tours d'angle a reçu sa forme définitive en Afrique, le souci des détails et la représentation en quatre tableaux des différents aspects d'un domaine pose le problème de l'intérêt documentaire de ce type de représentation. La mosaïque de Tolède est-elle le reflet d'une réalité objective, et donc hispanique? Sans doute la réponse doit-elle être en grande partie affirmative, bien qu'il faille évidemment demeurer prudent. Villa urbana,

(18) Sarnowski (T.), Op. cit, 1978, p. 74-75. 
représentation du maître et de sa famille, temple et jardin, pars rustica sont davantage les reflets d'un grand domaine éclaté du Bas-Empire ( ${ }^{19}$ ), que l'illustration d'imaginaires paysages idylliques. La luxueuse construction principale, mêlant galerie de façade, tours d'angle et péristyle, toits peints, suggère une villa seigneuriale de type aulique. Les murs d'enceinte enserrant la plupart des constructions évoquent des ensembles de fonctions différentes et pouvant être séparés géographiquement. Enfin, si les thermes ne sont pas représentés d'une manière appropriée, le thème de l'eau est suffisamment général pour contenir leur évocation d'une façon allusive, la place étant comptée. Mais le dernier panneau est aussi, et plus certainement, en relation avec une activité de commerce et de batellerie que l'on pourrait relier à la production agricole du domaine, laquelle transparaît aussi à travers les attributs des saisons ou la splendide tresse végétale et fruitière qui entoure 1 "emblema central. Il n'est pas exclu qu'un transport, par le Tage, ait pu concrètement s'étendre de la vega de Tolède jusqu'à l'océan: on sait combien, dans l'Antiquité, le fleuve était utilisé dans sa partie basse $\left({ }^{2 u}\right)$; on sait aussi qu'en 1581, grâce à des chemins de halage, il était encore navigable entre Lisbonne et Tolède $\left({ }^{21}\right)$ et que son module est suffisant en toute saison $\left({ }^{22}\right)$. Une telle liaison production-transport expliquerait les différentes représentations marines ou nautiques de la mosaïque des Quatre Saisons et celles du bassin octogonal, renforçant leur cohésion interne et leur mise en valeur mutuelle.

Toutes ces correspondances sont-elles suffisantes pour affirmer que nous sommes là en présence d'une illustration précise d'un

$\left.{ }^{19}\right)$ g о в в в (J.-G.), Les villas hispano-romaines (Op. cit. ), 1979, p. 111.

(20) garcia y вelino (A.), La navegabilidad de los ríos de la península Ibérica en la Antigüedad, dans «Investigación y Progreso», XVI, 1945, p. 115-122.

(21) м мов (F.), Diccionario geográfico-estadístico-histórico de España $y$ sus posesiones de Ultramar, tome XIV, Madrid, 1849, s.v. Tajo, p. 555-561 et stt. 558-559.

(22) masacho alaveda (V.), El régimen de los rios peninsulares, Barcelona, 1948, p. 496 et 507. 
domaine hispanique du Bas-Empire, représentative d'une réalité locale ? Certes non. Les quatre tableaux de la mosaïque des saisons mêlent l'imaginaire et le vrai, unissant des motifs qui sont autant de créations propres du mosaïste (cf. le propriétaire assis et son entourage...) que des emprunts à des sujets tirés du répertoire (cf. les éléments privilégiés d'architecture, les symboles de la navigation marchande...), l'important étant de rendre, avec le tout, l'image recherchée par le propriétaire d'une réalité qui soit à la fois typique et personnalisée.

C'est là, en effet, que réside le dernier point important. Le désir de concret du dominus oblige l'artiste à se rapprocher, au moins partiellement, d'un certain état de la réalité locale, et il apparaît bien par ailleurs que la mosaïque qui décore les riches habitations seigneuriales des IIle et IVe siècles présente d'autres fonctions que celles de simple ornement. Manifestation de la volonté personnelle d'un possessor orgueilleux, reflet de sa puissance sociale et économique, le pavement de Tolède participe aussi à l'illustration d'une idéologie propre à l'ensemble des grands propriétaires fonciers du Bas-Empire. Les deux galeries à colonnes de la villa urbana, bien en vue entre deux tours d'angle ou formant un demi-cercle exhaussé, dotées de proportions hors du réel, sont autant de symboles du pouvoir, expression par excellence de la richesse et de la romanité d'un propriétaire qui, pour apparaître à son avantage, trône dans ses jardins, entre sa famille et son temple privé, cumulant l'image du seigneur, du philosophe et de l'esthète. Unissant saisons, productions agricoles, vues du domaine, activités marchandes ou piscicoles (1 'emblema central est à sa façon un vivier), exaltant la puissance économique, sociale et spirituelle du maître, l'ouverture sur la mer (et donc sur Rome et sur l'Empire), la mosaïque tolédane participe pleinement au programme iconographique développé à l'usage des propriétaires de latifundia romains $\mathrm{i}^{23}$.

Elle nous apparaît aussi comme l'un des plus beaux exemples

(23) grabar (A.), Programme iconographique à Гusage des propriétaires de latifundia romains, dans «Les Cahiers de l'Archéologie», XI-XII, 19601962, p. 394-395. 
hispaniques de la personnalisation des pavements durant le BasEmpire, et représente, pour l'Espagne, dans son esprit et toutes proportions gardées, l'équivalent du pavement plus tardif de Tabarka, en Afrique du Nord. La disposition des scènes figuratives en demi-cercles n'est d'ailleurs pas sans rappeler la forme du trifolium qui abritait la mosaïque tunisienne.

Enfin, si la petitesse relative des tableaux trouve son explication dans le souci du mosaïste de respecter au plus près les indications du carton qui lui sert de support, on ne peut qu'être frappé par la logique interne qui a présidé à l'élaboration de ce pavement d'apparat et par les choix délibérés qui ont conduit à harmoniser, en les faisant se compléter, les deux admirables mosaïques connues de la «Vega Baja» de Tolède. 


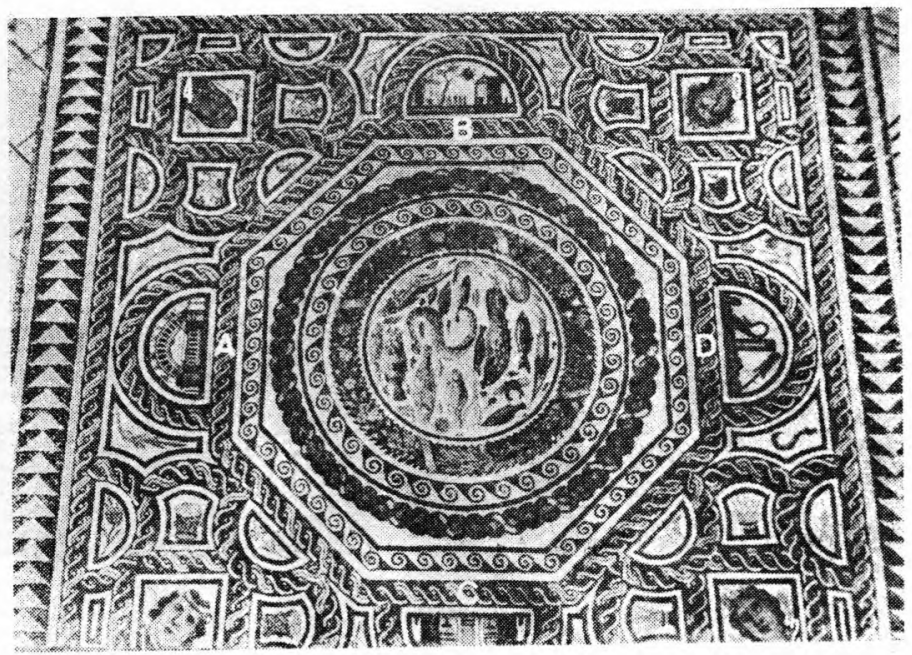

Fig. 1

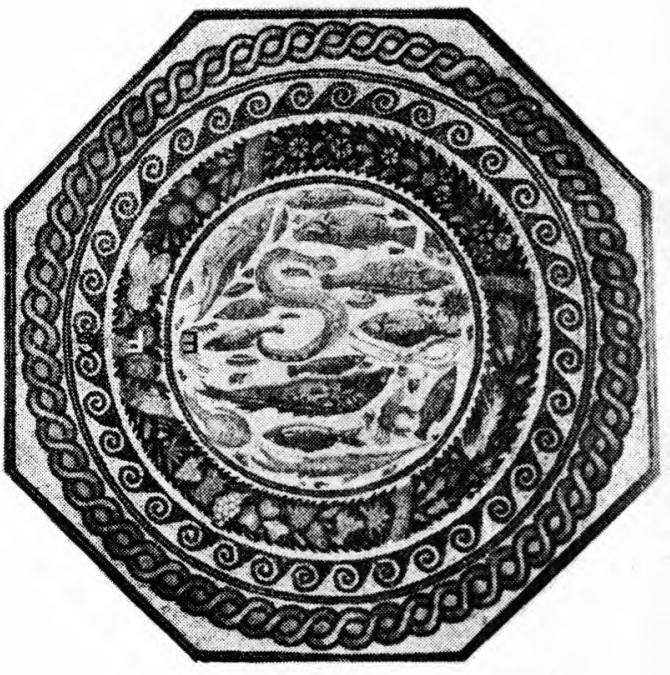

FIG. 2

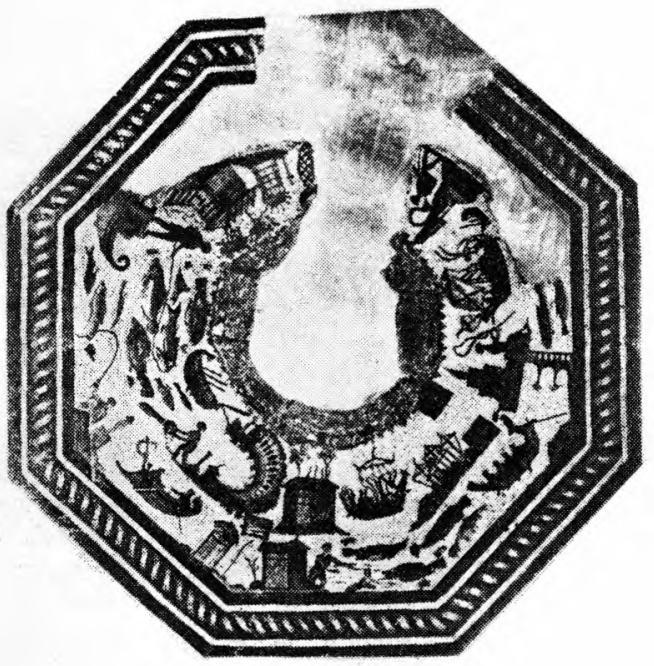

Fig. 3

Fig. 1 - Mosaïque des Quatre Saisons: vue d'ensemble et schéma de composition de la partie figurée; Fig. 2: Mosaïque des Quatre Saisons: l'octogone central: emblema marin et couronnes diverses; Fig. 3: Mosaïque du Bassin: vue d'ensemble. 
Planche II

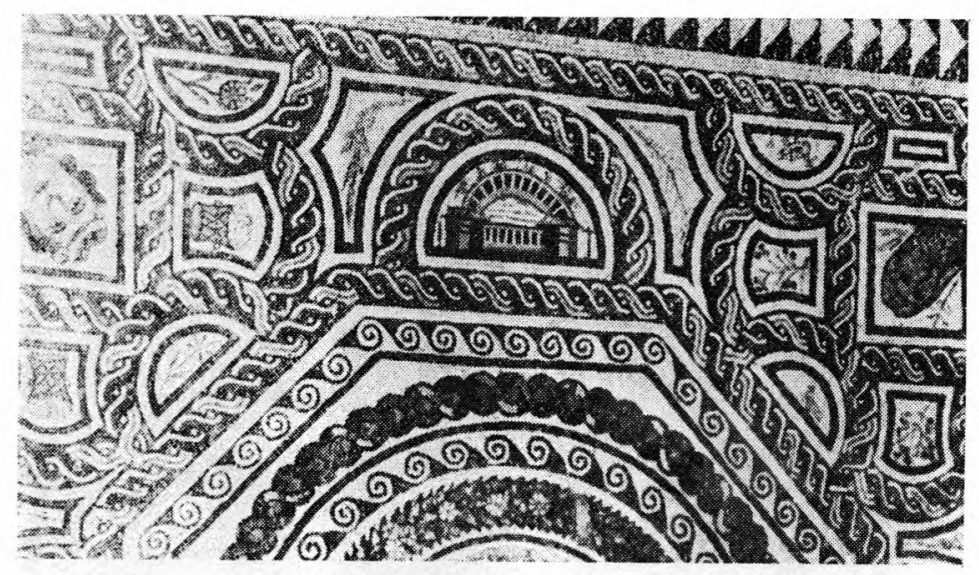

FIG. 1

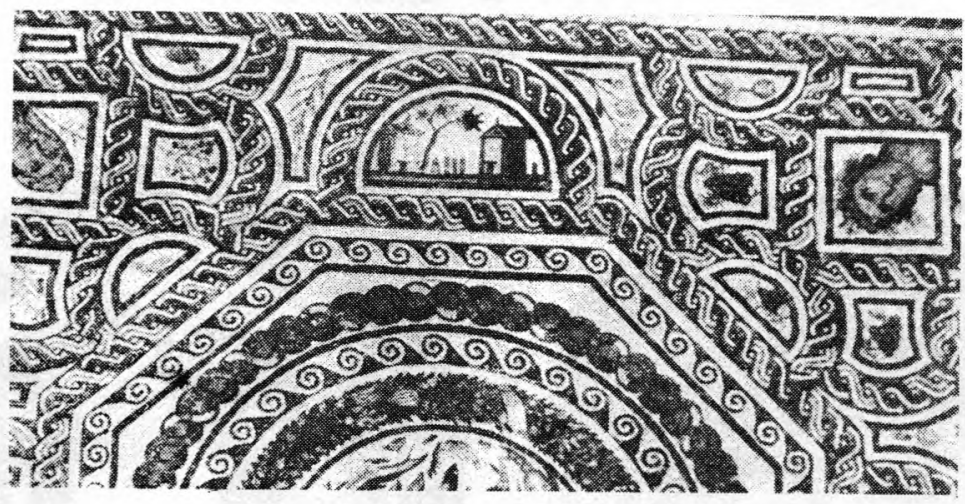

FIG. 2

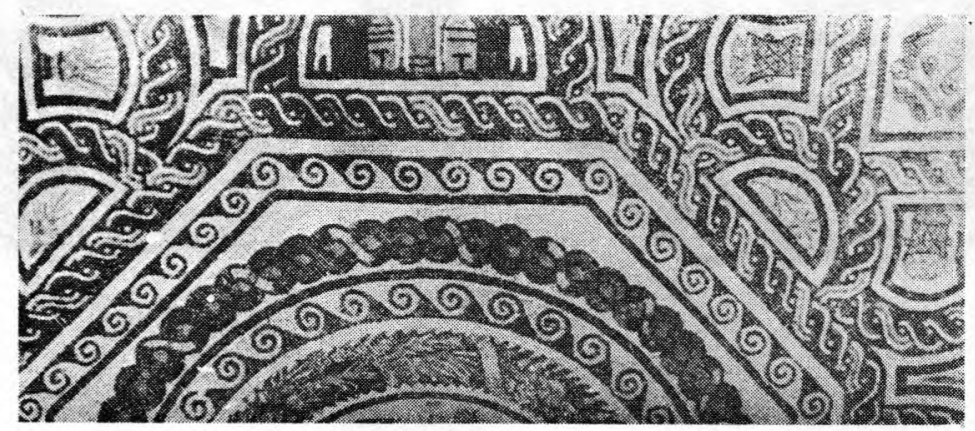

FIG. 3

Mosaïque des Quatre Saisons (détails): Fig. 1: la villa urbana; Fig. 2: les jardins; Fig. 3: la pars rustica. 


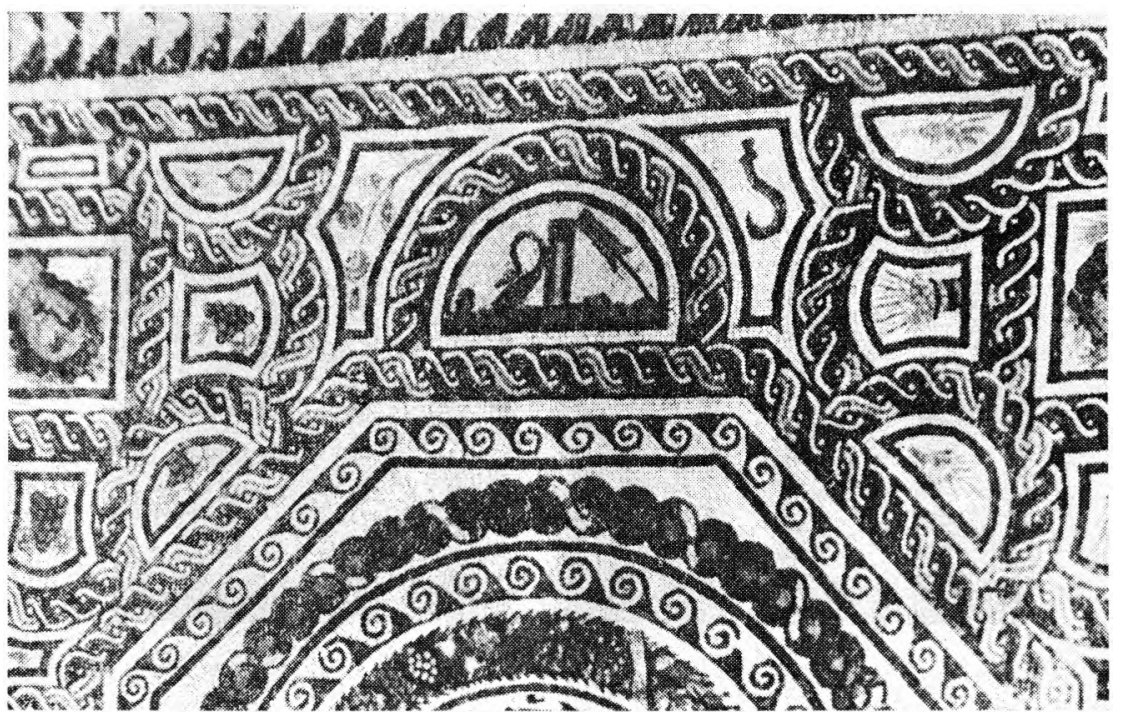

Fig. 1

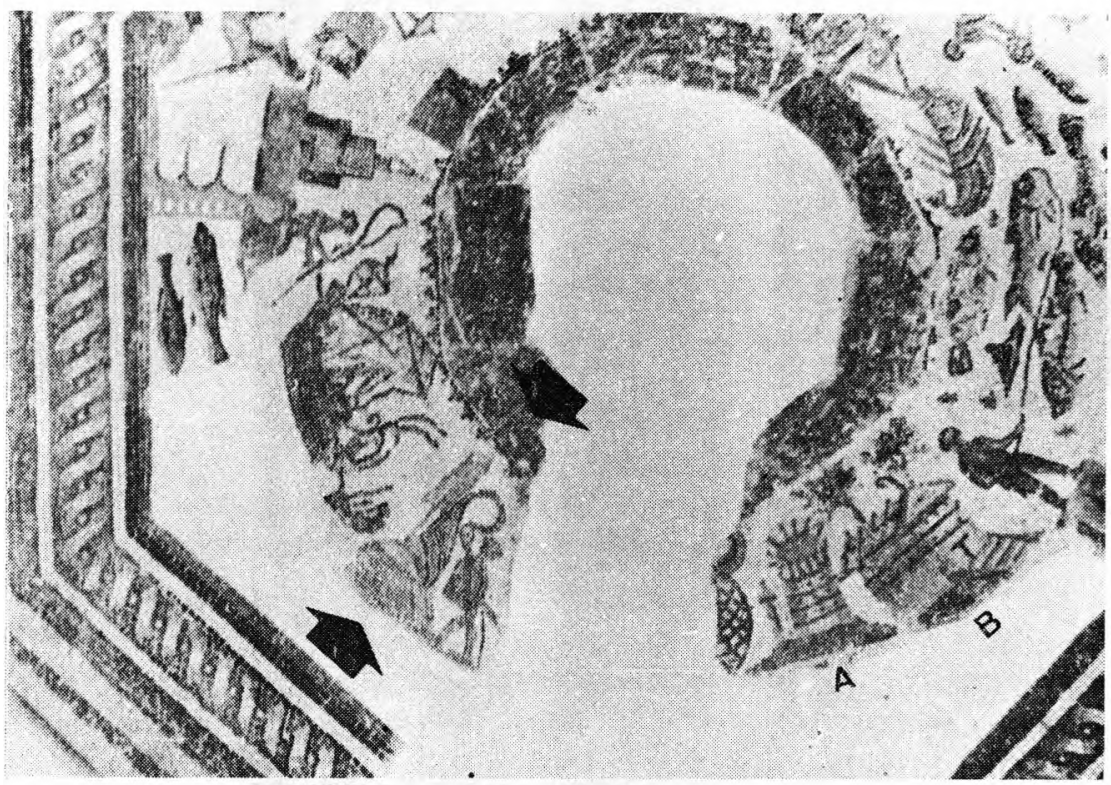

FIG. 2

Fig. 1: Mosaïque des Quatre Saisons (détail); le tableau nautique; Fig. 2: Mosaïque du Bassin (détail): le trophée nautique. 
PLANCHE IV

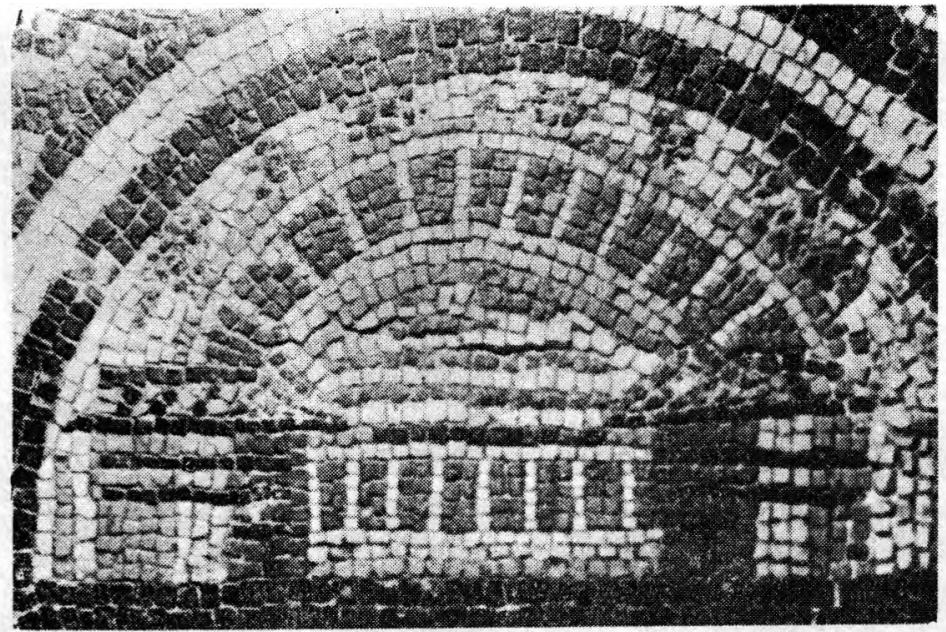

FIG. 1

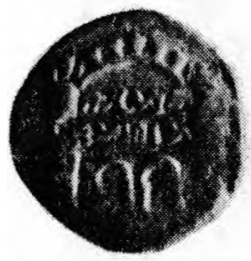

FIG. 3

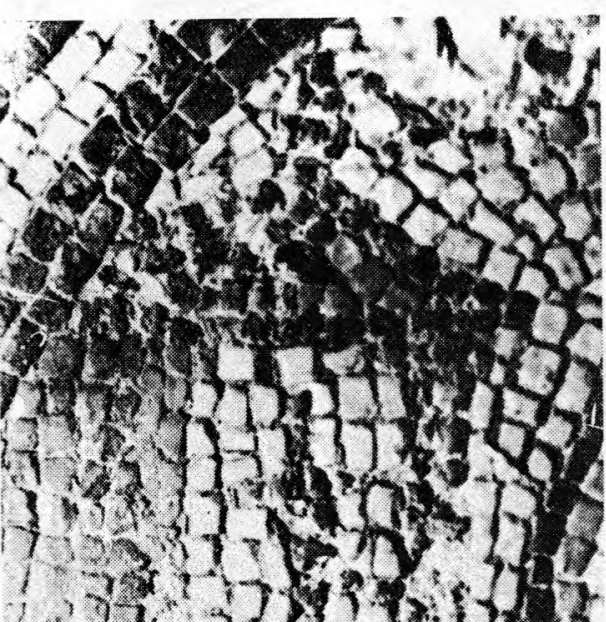

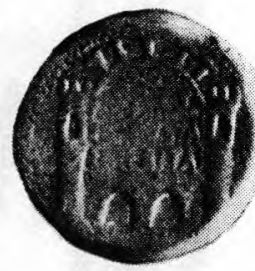

Fig. 4

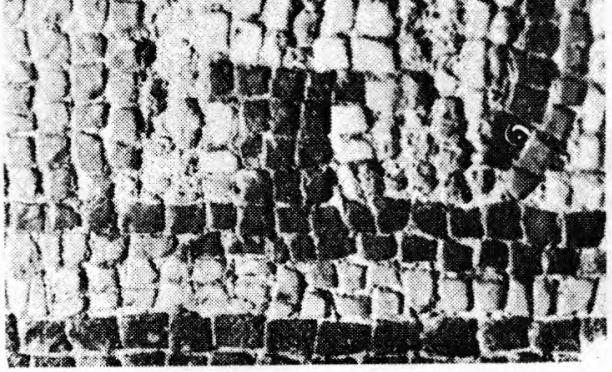

FIG. 2

Fig. 1: Mosaïque des Quatre Saisons: détail de la villa urbana; Fig 2: Mosaïque des Quatre Saisons: détail du temple du jardin; Fig. 3 et 4: Monnaies «à la porte de cité» d'Emerita Augusta. 